\title{
Improving door to needle time in neutropenic sepsis
}

\author{
Authors: Kieran Palmer, ${ }^{A}$ Emily Wang ${ }^{A}$ and Manab Mohanty ${ }^{A}$
}

\section{Aims}

Neutropenic sepsis is a potentially fatal complication for patients receiving systemic anti-cancer treatment (SACT). Mortality rates range between $2-21 \%$ among adults, and the use of aggressive intravenous antibiotics shows a significant reduction in both morbidity and mortality. The National Institute of Health and Care Excellence (NICE) guidance and local policy state that all patients who present to hospital as unwell post-chemotherapy should receive antibiotics within 1 hour. ${ }^{2}$ We aimed to reduce the presentation to antibiotics time among this group of patients and fundamentally improve patient safety and outcomes.

\section{Methods}

We identified all solid tumour and haematological oncology patients presenting to hospital as unwell post-chemotherapy using the electronic patient record (EPR) and referrals to the acute oncology service (AOS). We analysed compliance with NICE guidance and collected data over 6 months to establish a baseline. Firstly, we designed and implemented a robust standard operating procedure for neutropenic sepsis and the AOS delivered 6-monthly teaching to staff. Following this, to further improve our service, we introduced a cancer flagging icon within the EPR and on the emergency department (ED) whiteboard to immediately alert clinicians to patients who had recently undergone SACT. Posters were placed in the ED waiting room to educate patients for them to highlight to clinicians if they had also recently received treatment. We evaluated post-intervention data after both sets of interventions using the same criteria.

\section{Results and conclusions}

The implementation of both sets of interventions significantly improved the proportion of patients with suspected neutropenic sepsis who received antibiotics within 1 hour $(42 \%$ to $45 \%$ to $64 \%$ ). To further improve our results, we plan on introducing further neutropenic sepsis training to the trust induction and creating neutropenic sepsis grab packs within the ED

\section{Conflicts of interest}

None declared.

Authors: ${ }^{\mathrm{A}}$ Homerton University Hospital NHS Foundation Trust, London, UK

\section{References}

1 Herbst C, Naumann F, Kruse EB et al. Prophylactic antibiotics or G-CSF for the prevention of infections and improvement of survival in cancer patients undergoing chemotherapy. Cochrane Database Syst Rev 2009:CD007107.

2 Simmons T. Neutropenic sepsis: prevention and management of neutropenic sepsis in cancer patients. National Institute of Health and Care Excellence, 2012. www.nice.org.uk/guidance/cg151/ evidence/needs-assessment-pdf-188303583 [Accessed 15 October 2019]. 